\title{
Growth Laws and Self-Similar Growth Regimes of Coarsening Two-Dimensional Foams: Transition from Dry to Wet Limits
}

\author{
Ismael Fortuna, ${ }^{*}$ Gilberto L. Thomas, and Rita M. C. de Almeida ${ }^{\dagger}$ \\ Instituto de Física, Universidade Federal do Rio Grande do Sul, Caixa Postal 15051, 91501-970 Porto Alegre, RS Brazil
}

François Graner

"Polarité, Division et Morphogenèse," Laboratoire de Génétique et Biologie du Développement, Institut Curie, 26 rue d'Ulm, F-75248 Paris Cedex 05, France and Matière et Systèmes Complexes, Université Paris Diderot, CNRS UMR 7057, 10 rue Alice Domon et Léonie Duquet, F-75205 Paris Cedex 13, France

(Received 7 November 2011; published 14 June 2012)

\begin{abstract}
We study the topology and geometry of two-dimensional coarsening foam with an arbitrary liquid fraction. To interpolate between the dry limit described by von Neumann's law and the wet limit described by Marqusee's equation, the relevant bubble characteristics are the Plateau border radius and a new variable: the effective number of sides. We propose an equation for the individual bubble growth rate as the weighted sum of the growth through bubble-bubble interfaces and through bubble-Plateau border interfaces. The resulting prediction is successfully tested, without an adjustable parameter, using extensive bidimensional Potts model simulations. The simulations also show that a self-similar growth regime is observed at any liquid fraction, and they also determine how the average size growth exponent, side number distribution, and relative size distribution interpolate between the extreme limits. Applications include concentrated emulsions, grains in polycrystals, and other domains with coarsening that is driven by curvature.
\end{abstract}

DOI: 10.1103/PhysRevLett.108.248301

PACS numbers: $82.70 . \mathrm{Rr}, 83.80 . \mathrm{Iz}$

Liquid foam-namely, gas bubbles separated by a continuous liquid phase-are ubiquitous [1,2]. In floating foam such as beer head, ocean froth, or pollutant foam, the fraction $\phi$ of their volume that is occupied by the liquid decreases with height and varies from a dry foam at the top to a bubbly liquid at the foam-liquid interface.

Since pressure can differ from one bubble to another, gas slowly diffuses. Some bubbles disappear and, as no new bubbles are created, the average size increases. Foam coarsening is analogous to that of concentrated emulsions, grains in polycrystals, or two-phase domains where interface dynamics is driven by curvature. Its dynamics mainly depends on $\phi$, up to a material-specific time scale determined by the foam physicochemistry $[1,2]$.

Understanding foam coarsening requires two levels. First, the individual bubble growth law rules a bubble's growth rate according to its size or shape. This is a static geometry problem, and may be obtained analytically or by detailed bubble shape simulation. Second, the effect of such individual growth on the statistics of the foam, i.e., bubble size and topology distributions, requires statistical theories or extensive simulations.

In the very dry limit $\phi \rightarrow 0$, bubbles are polyhedra with thin curved faces that meet by three along thin lines called Plateau borders. Coarsening in that limit has been investigated experimentally, numerically, and theoretically in two dimensions (2D) [3-7] and later in three dimensions (3D) [8-14].
In the very wet limit $\phi \rightarrow 1$, bubbles are round, dispersed in the liquid, and far from each other, forming a "bubbly liquid" rather than a foam stricto sensu. Their coarsening follows Ostwald-Lifschitz-Slyozov-Wagner ripening in 3D [15,16], and Ostwald-Marqusee and Ostwald-YEGG in 2D [17,18].

In both limits, the foam eventually reaches a self-similar growth regime: statistical distributions of face numbers and relative sizes become invariant. The average size $\langle R\rangle$ grows in time as a power law, $\langle R\rangle \sim t^{\beta}$, with $\beta=1 / 2$ in the dry limit and $\beta=1 / 3$ in the wet one, due to the different underlying physical processes. The number $N$ of bubbles thus decreases as $t^{-2 \beta}$ in $2 \mathrm{D}$ and $t^{-3 \beta}$ in 3D. Intermediary liquid fraction regimes have been addressed in 3D experiments [19] and 2D simulations [20,21] but they still lack a unified theoretical description.

Focusing on the $2 \mathrm{D}$ case, we propose a growth law to interpolate for $0<\phi<1$, with two parameters (diffusion coefficients) determined in each limit. To our knowledge, there is no experimental study where $\phi$ is systematically varied and precisely measured, or even rigorously defined. So, comparing our predictions with experiments is not possible. Instead, we numerically simulate with the Potts model, which is suitable for large bubble numbers $[10,22-24]$. These simulations show that for any $\phi$, the side number and relative size distributions reach a selfsimilar growth regime where $\langle R\rangle$ grows as $t^{\beta}$. Values of $\beta$ interpolate between $1 / 2$ and $1 / 3$. 
In the 2D dry limit, gas diffuses through neighbor bubble walls due to the pressure difference between the bubbles related to wall curvature. The walls are curved because they meet at threefold vertices, forming equal angles of $2 \pi / 3$. Each vertex is responsible for a turn of $\pi / 3$ in the vector tangent to the bubble perimeter. Consequently, bubbles with five sides or fewer are convex, while bubbles with seven sides or more are concave, so that the walls' curvature plus a turn of $\pi / 3$ at each vertex sums to $2 \pi$ [1]. The resulting growth dynamics is von Neumann's law [4]

$$
\frac{d a^{i}}{d t}=-D_{d}\left(2 \pi-n^{i} \frac{\pi}{3}\right)=\frac{\pi D_{d}}{3}\left(n^{i}-6\right),
$$

where $a^{i}$ and $n^{i}$ are the area and the number of sides of the $i$ th bubble, respectively; $t$ is time and $D_{d}$ depends on the foam composition and is expressed in $\mathrm{m}^{2} \mathrm{~s}^{-1}$ as a diffusion coefficient. Remarkably, the rhs of Eq. (1) involves only the bubble's number of sides and not its size or shape. At any time, bubbles with $n^{i}<6$ shrink, while bubbles with $n^{i}>6$ grow. Since for topological reasons the average number of bubble sides is six [1,25], Eq. (1) is compatible with the gas volume conservation in the whole foam.

In the 2D wet limit, gas bubbles are dispersed in a liquid matrix. Dynamics is ruled by the pressure difference in the gas contained in a bubble and dissolved in the liquid, which is proportional to the inverse of the wall curvature radius, $R^{i}$. Marqusee's [17] growth law is as follows:

$$
\frac{d a^{i}}{d t}=2 \pi R^{i} \frac{d R^{i}}{d t}=2 \pi D_{w} f\left(R^{i}\right),
$$

with $D_{w}$ also a diffusion coefficient-like constant, and

$$
f\left(R^{i}\right)=\frac{R^{i}}{\xi} \frac{K_{1}\left(\frac{R^{i}}{\xi}\right)}{K_{0}\left(\frac{R^{i}}{\xi}\right)}\left[\frac{1}{R_{c}}-\frac{1}{R^{i}}\right],
$$

where $K_{j} \mathrm{~s}$ are $j$ th order modified Bessel functions of the second kind; $\xi$ is the screening length (roughly, the typical distance beyond which bubbles do not feel the influence of each other); and $R_{c}$ is the critical radius for which there is no growth, calculated by imposing total gas volume conservation, i.e., $\frac{d}{d t} \sum_{i} a^{i}=0$. At any given time, bubbles with radius smaller than $R_{c}$ lose gas, while those with radius larger than $R_{c}$ gain gas.

Interpolating between Eqs. (1) and (2) seems difficult because they use very different variables: the number of sides $n^{i}$ and the radius $R^{i}$. However, both equations involve the product of curvature times length (i.e., angle, Fig. 1) of the interfaces through which the gas diffuses. We propose that for any $\phi$ the bubble growth rate is simply the superposition of growth through the interfaces shared either directly with other bubbles or with Plateau borders. It can thus be calculated as the weighted average of Eqs. (1) and (2). The weights are fractions of $2 \pi$ angle carried by dry or wet parts of the $i$ th bubble perimeter, $\Theta_{d}^{i}$ or $\Theta_{w}^{i}$, which are sums of angles $\theta_{d}$ or $\theta_{w}$ carried,

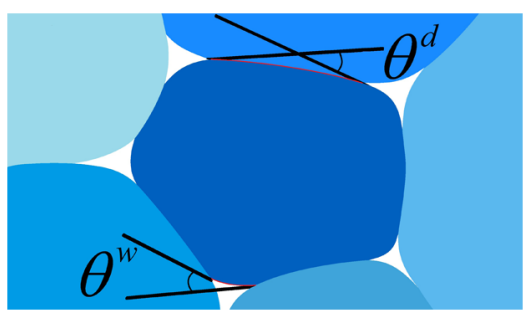

FIG. 1 (color online). Angles $\theta_{d}$ or $\theta_{w}$ measure the change in direction carried by a dry or wet interface. Here $n_{\text {eff }}$ is slightly larger than 5, the bubble is rather dry, $\Theta_{d}>\Theta_{w}, R_{w}<R$. Each white region represents a Plateau border and is simulated as several liquid drops (not drawn here).

respectively, by each dry or wet interfaces of the bubble (Fig. 1), such that $\Theta_{d}^{i}+\Theta_{w}^{i}=2 \pi$. We now detail how to perform this linear superposition, which turns out to be unexpectedly successful for all values of $\phi$.

In dry foam, at each vertex $\theta_{d}=\pi / 3$, so that $\Theta_{d}^{i}=$ $n^{i} \pi / 3$. In wet foam, $\Theta_{w}=2 \pi$. To interpolate, we characterize the bubble $i$ by its effective number of sides defined for any $\phi$ as follows:

$$
n_{\mathrm{eff}}^{i}=\frac{3}{\pi} \Theta_{w}^{i}=6-\frac{3}{\pi} \Theta_{d}^{i} .
$$

Although $n_{\text {eff }}^{i}$ conveys no more information than $\Theta_{d}$ or $\Theta_{w}$, its value is intuitive: $n_{\mathrm{eff}}^{i}=n^{i}$ for a polygonal dry bubble, $n_{\text {eff }}^{i}=6$ for a circular wet bubble, and $n_{\text {eff }}^{i} / 6$ is exactly the fraction of $2 \pi$ carried by the wet interfaces. Similarly, we characterize the bubble $i$ by the curvature radius $R_{w}^{i}$ of its Plateau borders, $R_{w}^{i}=0$ for a dry bubble, and $R_{w}^{i}=R^{i}$ for a wet bubble. Using $n_{\mathrm{eff}}^{i}$ and $R_{w}^{i}$ we can weight Eqs. (1) and (2) to interpolate for any $\phi$,

$$
\frac{d a^{i}}{d t}=\frac{\pi}{3}\left[D_{d}\left(n_{\mathrm{eff}}^{i}-6\right)+D_{w} n_{\mathrm{eff}}^{i} f\left(R_{w}^{i}\right)\right]
$$

To test Eq. (5) we have implemented extensive Potts model simulations in the spirit of Ref. [10]. Like in experimental pictures, a simulation represents gas bubbles and liquid phase as connected regions on a square lattice of pixels. To ensure that liquid is evenly distributed between Plateau borders (Fig. 1) and that $\phi$ is conserved, we represent the liquid phase as a fixed number of tiny "liquid drops" of fixed area, and each Plateau border contains several of these drops. Each interface between the gas bubbles represents a thin film made of two gas-liquid interfaces that mildly repel each other. We then assign to the interfacial energy between the gas bubbles a value $(2-\varepsilon)$ times the interfacial energy between the gas bubble and the liquid drop. Experimentally, in soap foam, $\varepsilon$ is typically of order $10^{-3}$. Its precise value is not crucial in what follows, as long as $\varepsilon \ll 1$ (and $\varepsilon>0$ to avoid numerical instabilities). Here we choose $\varepsilon=0.01$ as a compromise between realism and computing speed. Since drops are free to cluster together, we assign to drop-drop 
interfaces an energy $10^{3}$-times smaller. The liquid is sucked in the Plateau borders (for reviews see Refs. [1,26]).

There are $\mathcal{N}=8944^{2}$ sites; each site $s$ is assigned a label, $S(s)$. There are $N$ bubbles $(S=1$ to $N)$ and $N_{w}$ drops $\left(S=N+1\right.$ to $\left.N+N_{w}\right)$, assigned with a type $\tau=1$ or 0 , respectively. A configuration has an energy

$E=\sum_{s=1}^{\mathcal{N}} \sum_{s^{\prime}=1}^{36} J\left(\tau, \tau^{\prime}\right)\left[1-\delta_{S, S^{\prime}}\right]+\lambda \sum_{S=N+1}^{N+N_{w}}\left[a(S)-a_{t}\right]^{2}$,

where $s^{\prime}$ stands for the sum over the first 36 neighbors of the site $s$, to avoid pinning to the grid [27] and to extend the range of the disjoining pressure up to three pixels; $S$ and $S^{\prime}$ are the labels of sites $s$ and $s^{\prime}$, respectively; $\delta$ is the Kronecker symbol; $\tau$ and $\tau^{\prime}$ are the types of $S$ and $S^{\prime}$, respectively; $J(1,1)=1.99 J(1,0), \quad J(1,0)=J(0,1)=0.7$, and $J(0,0)=0.001$ are the interfacial energies; $a(S)$ is the drop current area $S ; a_{t}=8$ pixels is the drop target area; and $\lambda=9$ penalizes any deviation from $a_{t}$.

Simulations begin with $N_{0}=2 \times 10^{5}$ gas bubbles randomly dispersed over the grid, with smooth interfaces and a normal distribution of areas around the average $\frac{\mathcal{N}}{N_{0}}$. For $\phi>0$, initially each vertex contains at least one drop. The total number of drops and the initial average area of gas bubbles are set according to the desired $\phi$. The simulation dynamics follows the Monte Carlo method. We randomly choose a site, temporarily change its label to the value of one of its neighbors, and calculate the change in energy $\Delta E$. If $\Delta E \leq 0$, this relabeling is accepted. If $\Delta E>0$, the change is accepted with probability $\exp (-\Delta E / T)$, where $T$ is the fluctuation allowance, here taken as $T=3$ to escape possible metastable states.

We measure the diffusion coefficients as follows. We first perform a simulation in the dry limit $\phi=0$. Plotting the rhs and lhs of Eq. (1) determines by linear regression $D_{d}=1.68$. We then perform a simulation in the wet limit $\phi=0.9$. Plotting the rhs and lhs of Eq. (2) determines by linear regression $D_{w}=0.78$. With these parameters, together with area and wet and dry perimeters for each bubble from the simulations, Eq. (5) predicts without any adjustable parameter each bubble's growth for any intermediary $\phi$.

We measure the angles on a square grid as follows. The simulation is halted. We add over all of the dry interfacial pixels of bubble $i$ the probability that it will grow (or shrink) over (yielding to) other bubbles, calculated by the Monte Carlo method. This determines the growth rate of bubble $i$ through its dry interfaces, $G_{d}^{i}$, and thus $G_{d}^{i}=$ $-D_{d} \Theta_{d}^{i}$. We then obtain $\Theta_{w}^{i}=2 \pi-\Theta_{d}^{i}$ and $R_{w}^{i}=\frac{P_{w}^{i}}{\Theta_{w}^{i}}$ where $P_{w}^{i}$ is the wet interface length. During this measure, no change is performed. The simulation then resumes.

We run simulations with $\phi=0,0.02,0.06,0.18,0.36$, $0.54,0.72$, and 0.90 . For any $\phi$, the evolution of the gas bubble number has a power law behavior [Fig. 2(a)],

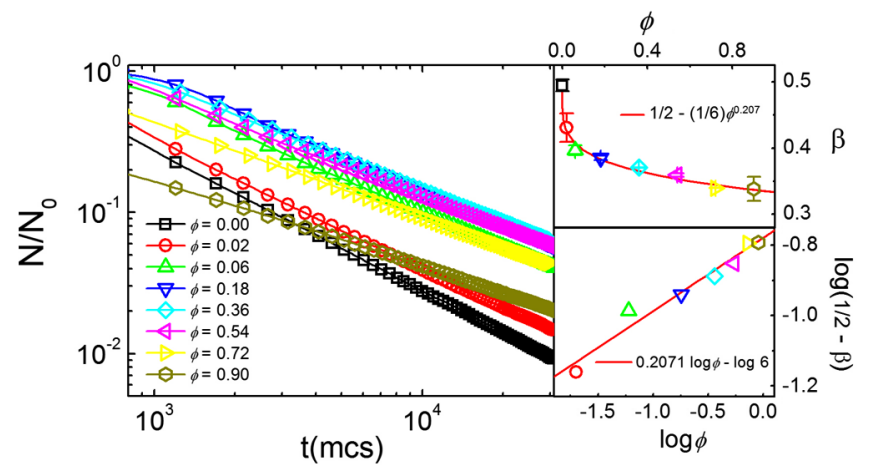

FIG. 2 (color online). (a) Evolution of the number of gas bubbles for different values of $\phi$, in $\log -\log$ plot. $(b, c)$ Power law exponent $\beta$ versus $\phi$, in (b) linear and (c) $\log$-log scales. The red line is $\beta=1 / 2-\phi^{0.207} / 6$.

which is compatible with self-similar growth regimes. Figures 2(b) and 2(c) show that the power law exponent $\beta$ varies as $\beta(\phi) \approx 1 / 2-\phi^{0.2} / 6$. Thus, $\beta(\phi)$ decreases continuously from $1 / 2$ to $1 / 3$, the expected limit values, with $d \beta / d \phi$ diverging at $\phi \rightarrow 0$. It would be interesting to explain theoretically this variation of $\beta$ with $\phi$.

Figure 3 shows snapshots for different $\phi$ after 20000 Monte Carlo steps (MCS). The liquid accumulates at the vertices for small $\phi$, and as it increases, liquid also goes between the bubbles. The ratio $d / \xi$, where $d$ is the typical distance between the bubbles, estimated as $d=$ $2(\sqrt{\mathcal{N} /(N \pi)}-\sqrt{\langle a\rangle / \pi})$, and $\xi$, the screening length [Eq. (3)], increases with $\phi$ (Fig. 1 of Ref. [28]). At $\phi=0.54, d / \xi>1$, and at $\phi=0.90$, the bubbles are not touching each other.

Figure 4 presents the average area growth rate of gas bubbles versus $R_{w}$, and in the insets the distribution function of $R_{w} /\left\langle R_{w}\right\rangle$. The superposition of plots taken at different times indicates a self-similar growth regime. The agreement with the theoretical prediction [Eq. (5)] is excellent. For $\phi=0$, all bubbles have $R_{w}=0$ and this plot does not convey any information. For $\phi=0.02$ and 0.06 the noise arises from measuring the curvature radius of the Plateau borders. For different values of $R_{w}$, distributions

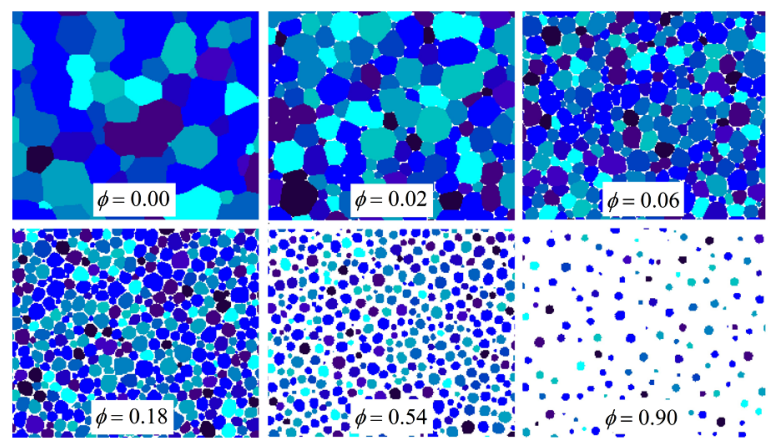

FIG. 3 (color online). Snapshots after $20000 \mathrm{MCS}$ for $\phi=0$, $0.02,0.06,0.18,0.54$, and 0.90 . 


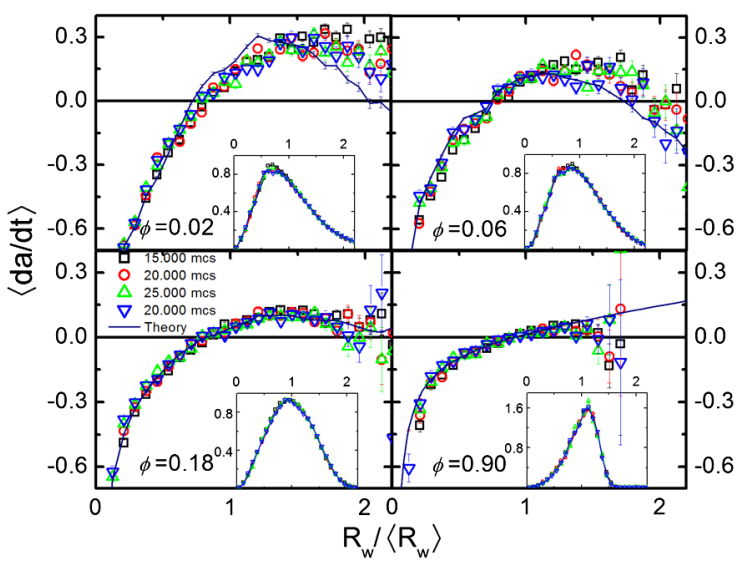

FIG. 4 (color online). Gas bubble growth rate versus normalized radius of Plateau borders, $R_{w} /\left\langle R_{w}\right\rangle$, at different times of the self-similar growth regime for $\phi=0.02$ to 0.90 . Insets: distribution function of $R_{w} /\left\langle R_{w}\right\rangle$. (See also Fig. 6 of [28].)

of the bubble growth rates are presented in Fig. 2 of Ref. [28].

Figure 5 presents the average area growth rate of gas bubbles versus the effective number of sides, $n_{\text {eff }}$. Again, the agreement with the theoretical prediction [Eq. (5)] is excellent. For $\phi=0.90$, all bubbles have $n_{\text {eff }}=6$ and this plot does not convey any information. For different values of $n_{\text {eff }}$, the distribution functions of the bubble growth rates are plotted in Fig. 3 of Ref. [28]. The plots of growth rates versus $n_{\text {eff }} /\left\langle n_{\text {eff }}\right\rangle$ (Fig. 4 of Ref. [28]) and versus $R_{w} /\left\langle R_{w}\right\rangle$ (Fig. 5 of Ref. [28]) discriminate the relative contributions of dry or wet interfaces to the growth.

In conclusion, we propose an equation for the average growth rate of bubbles in foam of arbitrary liquid fraction as the weighted sum of the growth through bubble-bubble interfaces (von Neumann's law), and growth through bubble-Plateau border interfaces (Marqusee's equation).

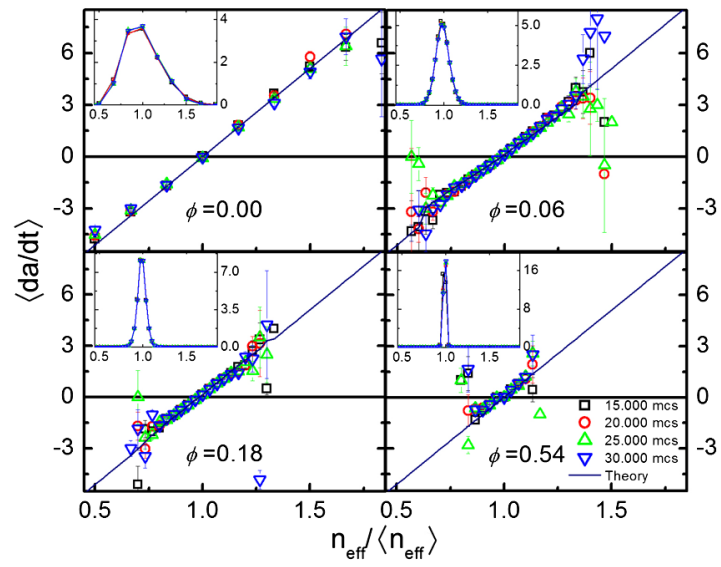

FIG. 5 (color online). Gas bubble growth rate versus $n_{\text {eff }} /\left\langle n_{\text {eff }}\right\rangle$ at different times of the self-similar growth regime for $\phi=0$ to 0.54 . Insets: distribution function of $n_{\text {eff }} /\left\langle n_{\text {eff }}\right\rangle$. (See also Fig. 7 of [28].)
The equation agrees very well with extensive 2D Potts model simulations. It would be interesting to check whether 2D foam experiments also follow these growth equations. Extending these simulations to 3D should not be a problem; as in 2D, Laplace's law, which depends on the curvatures of bubble walls, may be checked by measuring growth probabilities on the 3D Potts model. Applications include concentrated emulsions, grains in polycrystals, and other domains with coarsening driven by curvature.

This work was partially supported by CNPq, CAPES, and FAPERGS, and initiated during R.d. A.'s and G. L. T.'s discussions with F. G. at the LSP/LIPhy, University of Grenoble.

*ismaelfortuna@gmail.com

†rita@if.ufrgs.br

Also at Instituto Nacional de Ciência e Tecnologia: Sistemas Complexos, Porto Alegrem RS, Brazil.

[1] I. Cantat, S. Cohen-Addad, F. Elias, F. Graner, R. Höhler, O. Pitois, F. Rouyer, and A. Saint-Jalmes, Les mousses: Structure et dynamique (Belin, Paris, 2010) [English version, Foams: Structure and Dynamics, edited by S. Cox (Oxford University Press, Oxford, to be published), ISBN 978-0-19-966289-0].

[2] D. Weaire and S. Hutzler, Physics of Foams (Oxford University Press, Oxford, 2001).

[3] J. Stavans, Rep. Prog. Phys. 56, 733 (1993).

[4] J. von Neumann, in Metal Interfaces, edited by R. Brick (ASM, Cleveland, 1952), p. 108.

[5] J. Stavans and J. A. Glazier, Phys. Rev. Lett. 62, 1318 (1989).

[6] V. Pignol, Ph.D. thesis, Institut National Polytechnique de Lorraine, 1996 (unpublished).

[7] J. R. Iglesias and R. M. C. de Almeida, Phys. Rev. A 43, 2763 (1991).

[8] F. Wakai, N. Enomoto, and H. Ogawa, Acta Mater. 48, 1297 (2000)

[9] C. E. Krill, III and L.-Q. Chen, Acta Mater. 50, 3059 (2002).

[10] G. L. Thomas, R. M. C. de Almeida, and F. Graner, Phys. Rev. E 74, 021407 (2006).

[11] J. Lambert, R. Mokso, I. Cantat, P. Cloetens, J. A. Glazier, F. Graner, and R. Delannay, Phys. Rev. Lett. 104, 248304 (2010).

[12] P. Streitenberger and D. Zöllner, Scr. Mater. 55, 461 (2006).

[13] S. Hilgenfeldt, A. M. Kraynik, D. A. Reinelt, and J. M. Sullivan, Europhys. Lett. 67, 484 (2004).

[14] R. McPherson and D. Srolovitz, Nature (London) 446, 1053 (2007).

[15] W. Ostwald, Principles of Inorganic Chemistry (Macmillan, London, 1902), p. 58; Grundriss der Allgemeinen Chemie (Macmillan, London, 1908), p. 96; The Scientific Foundations of Analytic Chemistry (Macmillan, London, 1908), 3rd ed., p. 22. 
[16] I. M. Lifschitz and V. V. Slyozov, Zh. Eksp. Teor. Fiz. 35, 479 (1958) [Sov. Phys. JETP 8, 331 (1959)]; C. Wagner, Z. Elektrochem. 65, 581 (1961)

[17] J. A. Marqusee, J. Chem. Phys. 81, 976 (1984).

[18] J. H. Yao, K. R. Elder, H. Guo, and M. Grant, Phys. Rev. B 45, 8173 (1992).

[19] J. Lambert, I. Cantat, R. Delannay, R. Mokso, P. Cloetens, J. A. Glazier, and F. Graner, Phys. Rev. Lett. 99, 058304 (2007).

[20] F. Bolton and D. Weaire, Philos. Mag. B 65, 473 (1992).

[21] S. Hutzler, D. Weaire, and F. Bolton, Philos. Mag. B 71, 277 (1995).

[22] M. P. Anderson, G. S. Grest, and D. J. Srolovitz, Philos. Mag. B 59, 293 (1989).
[23] J.A. Glazier, M.P. Anderson, and G.S. Grest, Philos. Mag. B 62, 615 (1990).

[24] D. Zöllner and P. Streitenberger, Scr. Mater. 54, 1697 (2006).

[25] W. C. Graustein, Ann. Math. 32, 149 (1931).

[26] V. Bergeron, J. Phys. Condens. Matter 11, R215 (1999).

[27] E. A. Holm, J. A. Glazier, D. J. Srolovitz, and G. S. Grest, Phys. Rev. A 43, 2662 (1991).

[28] See Supplemental Material at http://link.aps.org/ supplemental/10.1103/PhysRevLett.108.248301 for plots of bubble distance to screening length ratio versus liquid fraction, and for growth probability distributions and growth rates versus effective side numbers and Plateau border radius. 